\title{
Implementing a thoracic enhanced recovery with ambulation after surgery program: key aspects and challenges
}

\author{
Marissa A. Mayor, Sandeep J. Khandhar, Joby Chandy, Hiran C. Fernando \\ Inova Cardiac and Thoracic Surgery, Department of Surgery, Inova Fairfax Medical Campus, Falls Church, Virginia, USA \\ Contributions: (I) Conception and design: All authors; (II) Administrative support: SJ Khandhar; (III) Provision of study materials or patients: \\ SJ Khandhar; (IV) Collection and assembly of data: SJ Khandhar; (V) Data analysis and interpretation: All authors; (VI) Manuscript writing: All \\ authors; (VII) Final approval of manuscript: All authors. \\ Correspondence to: Hiran C. Fernando, MBBS. Inova Cardiac and Thoracic Surgery, Department of Surgery, Inova Fairfax Medical Campus, 3300 \\ Gallows Road, Falls Church, Virginia 22042, USA. Email: Hiran.Fernando@inova.org.
}

\begin{abstract}
Enhanced recovery after surgery (ERAS) protocols aim to improve operative outcomes by focusing on perioperative care, including early mobilization, limitation of narcotics, and maintenance of fluid balance. We implemented a T-ERA ${ }^{A} S$ (Thoracic-Early Recovery with Ambulation After Surgery) protocol which focused on early ambulation, with the rationale that a patient's mobility may be a reproducible and measurable metric for their overall status-pain control, respiratory function, cardiac function, and patient satisfaction. We set a benchmark distance of 250 feet for our early ambulation goal and redefined "early" as within the first hour post extubation. We describe some of the major aspects to our program as well as some of the challenges and successes during our 8-year experience following the implementation of this program.
\end{abstract}

Keywords: Enhanced recovery after surgery (ERAS); enhanced recovery; fast track; video-assisted thoracic surgery (VATS); thoracic surgery; perioperative management; early ambulation

Submitted Jul 09, 2018. Accepted for publication Oct 29, 2018.

doi: $10.21037 /$ jtd.2018.10.106

View this article at: http://dx.doi.org/10.21037/jtd.2018.10.106

\section{Introduction}

Enhanced recovery after surgery (ERAS) protocols aim to improve operative outcomes through evidence-based perioperative care (1). The ERAS Society has published guidelines for procedure specific recovery including colorectal, gastric, and gynecologic surgeries (2). The common principles include minimization of preoperative risk (smoking cessation, nutritional support, exercise), limitation of narcotics, maintenance of fluid balance, and early mobilization. Additional challenges unique to thoracic surgery include the physiologic insult caused by one lung ventilation intraoperatively and pain from intercostal incisions limiting respiratory effort. There are currently no consensus guidelines from the ERAS group for general thoracic surgery; however, several groups have published their initial experiences with enhanced recovery protocols (3-10). Common elements include preoperative optimization with smoking cessation and preoperative exercise regimens, intraoperative care modifications with a focus on minimally invasive techniques, drain limitation, and long term narcotic avoidance. A systematic meta-analysis of ERAS for lung cancer surgery found that ERAS patients had significantly lower morbidity rates $(\mathrm{RR}=0.64, \mathrm{P}<0.001)$ than the control patients, although there was no significant difference in the in-hospital mortality (11). While the principles of ERAS for thoracic surgery are consistent with more general ERAS protocols, these reports demonstrate the wide variation in specific management guidelines at each institution. In particular, the post-operative "early mobility" goal is a generalized encouragement of mobility with no report of a quantitative assessment. Some institutions have a goal of mobilization to a chair on the operative or first postoperative day $(7,8,10)$ while others have ambulation as the goal, anywhere from the first post-operative day $(4,6,12)$ to the day of surgery $(3,13)$. 


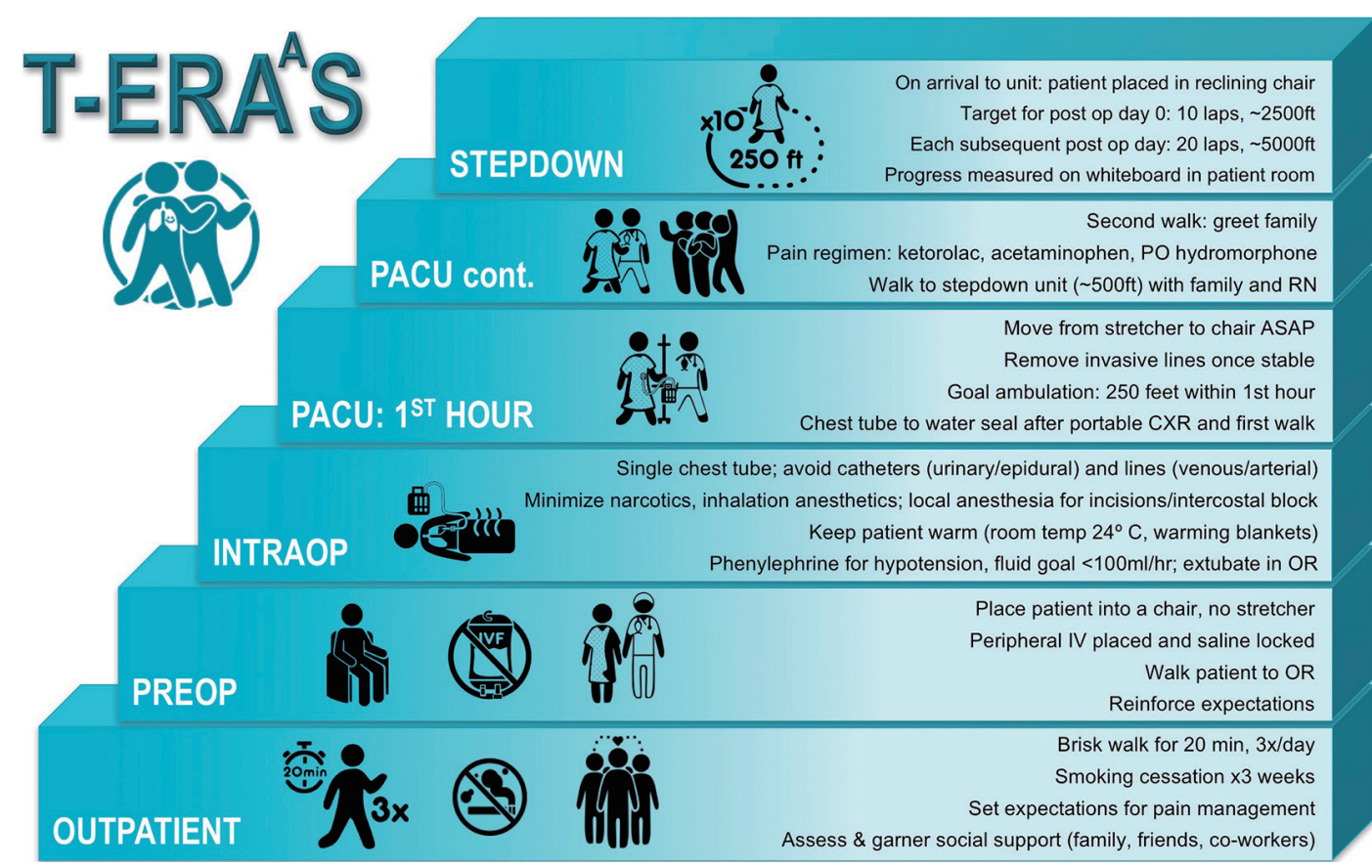

Figure 1 The T-ERA ${ }^{A} S$ protocol begins at the preoperative outpatient visit and focuses on ambulation. T-ERA ${ }^{A} S$, Thoracic-Early Recovery with Ambulation After Surgery.

We previously implemented a T-ERA ${ }^{\mathrm{A}} \mathrm{S}$ (Thoracic-Early Recovery with Ambulation After Surgery) protocol which focused on early ambulation that begins in the outpatient setting and continues in the post-operative anesthesia care unit (PACU) on the day of surgery (Figure 1). Our rationale for focusing on ambulation was that a patient's mobility is a tangible, reproducible, and measurable endpoint around which other more subjective markers should improve, namely, pain control, respiratory function, cardiac function, and patient satisfaction. There are no programs to our knowledge whose primary focus was transforming the time and distance patients ambulate in the immediate postoperative period. We recently published our results from this program with a focus on a readily comparable subset of patients, specifically patients undergoing thoracoscopic lobectomy (14). The report includes 304 patients who underwent video-assisted thoracic surgery (VATS) lobectomy, and results were compared to 100 patients who also underwent VATS lobectomy, but were treated prior to the implementation of T-ERA ${ }^{A}$ S. Our median length of stay was 1 day compared to 2 days before implementation $(\mathrm{P}<0.001)$. Pneumonia rates were also significantly reduced from $6 \%$ to $0.7 \%$ after implementation of T-ERA ${ }^{\mathrm{A}} \mathrm{S}$. After 8 years of developing this program, our post-surgical care is streamlined. Visitors to our post anesthesia care unit
(PACU) often remark on the unique atmosphere. The most striking scene is the patient who has just been wheeled from the operating room placed in a chair within several minutes of arrival, and ambulates down the hall less than one hour following extubation. Achievement of this goal required changing the culture of our entire team, from the presurgical and intraoperative phases, to the PACU and step down care. Here, we describe our current program as well as some of the challenges we have faced in its evolution.

\section{Culture change}

The success of T-ERA ${ }^{A} S$ was dependent on the full participation and "buy-in" of every health care professional involved in the care of the patient, from the preoperative office visit to the post-operative discharge, as well as the patient's support system, or "family". While expectations were set by the surgeon in the first preoperative visit, we found that it was mandatory to reinforce this in the immediate preoperative period. Our patients and their families are reminded by the surgical staff (including not only the surgeon and surgeon assistant/resident, but the preoperative nurse and operating team that although pain control is important, our number one priority is returning them to their pre-operative state or better. Successful 
ambulation facilitates clearing of secretions thereby minimizing the risks of pneumonia; improves vascular tone and third space fluid shifts thereby minimizing the risk of arrhythmias; decreases the incidence of lower extremity deep venous thromboses and subsequent pulmonary emboli; improves pain control; allows for a general sense of wellbeing and independence; and facilitates faster recovery. Setting this tone at the initial visit and reinforcing this idea during each of the phases below allows for the successful implementation of T-ERA ${ }^{A}$ S. Our general thoracic procedures take place in the Heart and Vascular Institute of our medical center. This is a separate (but connected) building to the main hospital and has six operating suites and four post-anesthesia care unit (PACU) beds. Only cardiac, thoracic, and vascular cases are performed in these suites. General thoracic cases are recovered in the PACU, and then can walk from the PACU to their hospital beds in our step-down unit which is on the same floor as the operating room and PACU. This relatively small sized unit with its consistent team has facilitated the change in culture and success of the T-ERA ${ }^{\mathrm{A} S}$ program for thoracic surgical patients (15).

\section{Preoperative phase}

Published ERAS protocols for thoracic surgery are consistent in encouragement of smoking cessation and ambulation. Smoking cessation has been demonstrated to significantly improve the morbidity of thoracic surgeries (16), although the optimal time point for cessation prior to surgery has yet to be established. Most ERAS studies do not report the success rate of smoking cessation prior to surgery. Preoperative exercise is also routinely encouraged across institutions. High intensity preoperative training regimens have been evaluated with randomized trials, however improvement in operative outcomes has still to be demonstrated (17-19). Our approach has been to require patients to take a brisk, $20 \mathrm{~min}$ walk at least three times daily. Smoking cessation is expected, and we require complete cessation at least 3 weeks prior to the date of operation. Family members are encouraged to join the patient in this endeavor. At this point, the expectations for pain control are set. Our management philosophyavoidance of complications and rapid return to full function-is described to the patient, including discussion of a minimally invasive approach, use of local anesthetic techniques, minimization of narcotic use, and a thorough explanation of the rationale for early, frequent, and ongoing ambulation. The family is enlisted in the effort to achieve these ambulation goals. As our program has developed, we have observed that the family is crucial to the patient's postoperative success.

The topic of chest-tube management is also introduced in the initial counseling meeting and how this may impact early discharge. If patients have adequate home support and have evidence of a possible air-leak, they are discharged with their chest-tube in place attached to a mini-atrium. On the day of surgery, the patient is placed into a chair, not a stretcher, further augmenting the idea of mobility and independence. An intravenous catheter is placed, but other lines-if needed-are placed after induction in the operating room. If no contraindications, patients are given 1,000 $\mathrm{mg}$ of oral acetaminophen. Every health care professional reiterates the ambulation philosophy and the family is once again reminded by the surgeon that ambulation and the avoidance of complications, rather than pain control is the main priority. Finally, the patient ambulates from the preoperative area to the operating room and is assisted onto the table. These steps prime the patient for a successful recovery by helping re-inforce the mind-set of early ambulation.

\section{Intraoperative phase}

In order to facilitate post-operative mobility, lines (including central or arterial) and catheters (urinary or epidural) are routinely avoided, if at all possible, in patients scheduled for thoracic surgery. Fluid restriction is achieved by delivering all fluids and medications through programmed infusion pumps with a goal of delivering less than $100 \mathrm{~mL} / \mathrm{hr}$. Hypotension is routinely treated with vasoactive medications such as phenylephrine and ephedrine. Induction of anesthesia is fairly routine with the use of propofol and a muscle relaxant such as rocuronium to aid with intubation. Short-acting narcotics such as fentanyl are used at the time of intubation but limited to 100-200 micrograms. Longer-acting intravenous narcotics such as hydromorphone are discouraged from intraoperative use. Ketorolac (30 mg IV) is given at the end of the surgical procedure after consultation with the surgeon and if the glomerular filtration rate is $>60$. Warmed air blankets are used to prevent hypothermia. Lower extremity sequential compression devices are used routinely. Over the last six years, different approaches to providing local or regional anesthesia have been employed, some as part of prospective studies on pain control. These strategies have included thoracic 
epidurals, serratus and paravertebral blocks, intercostal bupivacaine injections, intercostal infusions (On-Q), liposomal bupivacaine, and bupivacaine injection at the incisions. Usually, a single chest tube is placed to suction. The patient is routinely extubated prior to leaving the operating suite.

\section{PACU}

Immediately upon arrival to the PACU an attempt is made to move the patient from the stretcher to the chair. This is a marked difference from published ERAS protocols for thoracic surgery; in fact, we were able to find only a single program which reported a goal of mobilization to a chair prior to four hours post-surgery (8). If this is deemed unsafe by nursing staff, (e.g., if the patient is unable to mentate or the vitals are tenuous) then an attempt is made every 3-5 minutes thereafter. After this is achieved, an attempt at ambulation is performed within the first hour following arrival to the PACU, with a target goal of 250 feet. Again, this is a unique aspect of our program; few programs encourage ambulation on the day of operation $(3,6)$ with most waiting until the first post-operative day $(4,10)$. The PACU nurses routinely document time to ambulation and whether a full 250 feet is achieved. If the full 250 feet is not achieved, a comment is recorded by the PACU nurse, and repeated attempts are made until the target distance is met. During the initial implementation of this program, our surgeons were readily available in the immediate postoperative phase, and if there was any concern regarding the patient's ability to ambulate, or if the nurse felt that they would not be ready to walk within the first half an hour, a call was made to the surgeon. As our staff became more comfortable with the concept of walking immediately after the procedure, the time to ambulation after extubation has improved. In our reported series of 304 VATS lobectomy patients, $61.5 \%$ of patients achieved the target goal of 250 feet within 1 hour (14). Some degree of ambulation was achieved in $68.4 \%$ in 1 hour and $94.7 \%$ in 2 hours. The mean time to ambulation was 57.3 minutes. When we compared our early (years 2010-2012) to later experience (2014-2016) we saw significant improvements in several ambulation parameters. For instance the target of 250 feet in one hour was achieved in only $37.3 \%$ early on compared to $72 \%$ later on. Similarly the mean time to ambulation improved from 80.5 to 46.2 minutes with $28 \%$ of patients ambulating within 30 minutes of extubation in the most recent two years (14).
If patients' vitals are stable, any invasive monitoring lines are removed prior to transfer to the stepdown unit. Pain control in the PACU is achieved with ketorolac (if appropriate), acetaminophen, and if needed, oral hydromorphone. Intravenous narcotics are minimized and discouraged. A chest radiograph is obtained and reviewed. The chest tube is placed to waterseal after the chest radiograph is completed and ambulation of 250 feet has been successfully accomplished. When feasible, the patient is walked toward the family waiting room to greet their family rather than the family coming to visit the patient. When the patient is cleared, they will walk to the step down unit $(\sim 1,000$ feet) with their family and nurse in attendance.

\section{Care in the stepdown unit}

After their walk from the PACU, the patient is placed in a reclining chair. A set of checkboxes for goal of ambulation is written on their whiteboard. The goal is 10 laps $(2,500 \mathrm{ft})$ around the unit for operative day 0 , and 20 laps for each post-operative day to follow. The patient is usually started on a diet once awake in the PACU and intravenous fluids are not routinely administered post-operatively. A morning chest X-ray is taken. If there is no air-leak, drainage is appropriately serous, and no large residual space seen on chest X-ray, then the chest-tube will usually be removed on the first post-operative day, and the patient discharged. Post-pull chest $\mathrm{X}$-rays are not usually performed. If there is an air-leak or a moderate air-space present, then the patient will be discharged with their chest-tube in place, unless other co-morbidities exist. Using this strategy postoperative discharge at one day after VATS lobectomy, was achieved in $75 \%$ of patients compared to only $25.8 \%$ of patients prior to T-ERA ${ }^{\mathrm{A}} \mathrm{S}$ implementation. Thirty-three $(10.86 \%)$ of patients were discharged with chest-tubes in place and re-admissions have only been required in 15/304 (4.93\%) after VATS lobectomy (14).

\section{Conclusions}

With the concept of ERAS now widely accepted across many institutions, we found that the acceptance and implementation of many aspects of our T-ERA $\mathrm{S}$ program, including factors such as intraoperative modifications to analgesia, intra-operative fluid management as well as postoperative limitation of narcotics were relatively simple to make standard of care. An advantage in our center is that thoracic surgical operations take place in a relatively small 
unit, so there is consistency of care and shared goals are more achievable. Setting a quantifiable goal for ambulation and documenting outcomes helps orient nursing staff, patients and their family members. The focus of care becomes overall recovery with return to normal function and less about optimizing pain management. This may be more challenging to achieve in other health systems where there is less consistency in operating room and nursing staff. Additionally, patient's social and family support may not lend itself easily to implementing these goals. The collaboration between surgeon, advance practice providers, anesthesiologists, nursing, administration, and the family is crucial in the success of T-ERA ${ }^{\mathrm{A}} \mathrm{S}$.

Although using a minimally invasive approach facilitates implementation of T-ERA ${ }^{\mathrm{A}} \mathrm{S}$, this is by no means restricted to VATS lobectomy. During the six-year period that we reported on 304 VATS lobectomies, 1,172 patients in total were treated on our early ambulation protocol after thoracic operations. Currently we are evaluating the results of early ambulation after thoracotomy and are also prospectively evaluating early ambulation in patients undergoing esophageal surgery including esophagectomy. We believe that setting any ambulation goal is vital to achieving the goal of early recovery, and we are hopeful that others will be encouraged to adopt aspects of our program.

\section{Acknowledgements}

The authors thank Marcela L. Mayor for her artistic contribution to this paper.

\section{Footnote}

Conflicts of Interest: the authors have no conflicts of interest to declare.

\section{References}

1. Ljungqvist $O$, Scott $M, K C$ F. Enhanced recovery after surgery: A review. JAMA Surg 2017;152:292-8.

2. ERAS Society List of Guidelines Available online: http:// erassociety.org/guidelines/list-of-guidelines/

3. Muehling BM, Halter GL, Schelzig H, et al. Reduction of postoperative pulmonary complications after lung surgery using a fast track clinical pathway. Eur J Cardiothorac Surg 2008;34:174-80.

4. Sokouti M, Aghdam BA, Golzari SEJ, et al. A Comparative Study of Postoperative Pulmonary Complications
Using Fast Track Regimen and Conservative Analgesic Treatment: A Randomized Clinical Trial. Tanaffos 2011;10:12-9.

5. Abu Akar F, Chen Z, Yang C, et al. Enhanced recovery pathways in thoracic surgery: The Shanghai experience. J Thorac Dis 2018;10:S578-82.

6. Rogers LJ, Bleetman D, Messenger DE, et al. The impact of enhanced recovery after surgery (ERAS) protocol compliance on morbidity from resection for primary lung cancer. J Thorac Cardiovasc Surg 2018;155:1843-52.

7. Brunelli A, Thomas C, Dinesh P, et al. Enhanced recovery pathway versus standard care in patients undergoing videoassisted thoracoscopic lobectomy. J Thorac Cardiovasc Surg 2017;154:2084-90.

8. Hubert J, Bourdages-Pageau E, Garneau CA, et al. Enhanced recovery pathways in thoracic surgery: the Quebec experience. J Thorac Dis 2018;10:S583-S590.

9. Gonfiotti A, Viggiano D, Voltolini L, et al. Enhanced recovery after surgery and video-assisted thoracic surgery lobectomy: the Italian VATS Group* surgical protocol. J Thorac Dis 2018;10:S564-S570.

10. Martin LW, Sarosiek BM, Harrison MA, et al. Implementing a Thoracic Enhanced Recovery Program: Lessons Learned in the First Year. Ann Thorac Surg 2018;105:1597-604.

11. Li S, Zhou K, Che G, et al. Enhanced recovery programs in lung cancer surgery: Systematic review and metaanalysis of randomized controlled trials. Cancer Manag Res 2017;9:657-70.

12. Madani A, Fiore JF Jr, Wang Y, et al. An enhanced recovery pathway reduces duration of stay and complications after open pulmonary lobectomy. Surgery 2015;158:899-908; discussion 908-10.

13. Numan RC, Klomp HM, Li W, et al. A clinical audit in a multidisciplinary care path for thoracic surgery: An instrument for continuous quality improvement. Lung Cancer 2012;78:270-5.

14. Khandhar SJ, Schatz CL, Collins DT, et al. Thoracic enhanced recovery with ambulation after surgery: a 6-year experience. Eur J Cardiothorac Surg 2018;53:1192-8.

15. Schatz C. Enhanced Recovery in a Minimally Invasive Thoracic Surgery Program. AORN J 2015;102:482-92.

16. Mason DP, Subramanian S, Nowicki ER, et al. Impact of Smoking Cessation Before Resection of Lung Cancer: A Society of Thoracic Surgeons General Thoracic Surgery Database Study. Ann Thorac Surg 2009;88:362-70; discussion 370-1.

17. Licker M, Karenovics W, Diaper J, et al. Short-Term 
Preoperative High-Intensity Interval Training in Patients Awaiting Lung Cancer Surgery: A Randomized Controlled Trial. J Thorac Oncol 2017;12:323-33.

18. Pouwels S, Fiddelaers J, Teijink JA, et al. Preoperative exercise therapy in lung surgery patients: A systematic

Cite this article as: Mayor MA, Khandhar SJ, Chandy J, Fernando HC. Implementing a thoracic enhanced recovery with ambulation after surgery program: key aspects and challenges. J Thorac Dis 2018;10(Suppl 32):S3809-S3814. doi: 10.21037/ jtd.2018.10.106 review. Respir Med 2015;109:1495-504.

19. Huang J, Lai Y, Zhou X, et al. Short-term high-intensity rehabilitation in radically treated lung cancer: a threearmed randomized controlled trial. J Thorac Dis 2017;9:1919-29. 